\title{
Quality of life in patients treated by organ preservation surgery for early laryngeal carcinoma
}

This article was published in the following Dove Press journal:

Open Access Surgery

12 October 2012

Number of times this article has been viewed

\section{Eugenia Allegra \\ Teresa Franco \\ Serena Trapasso \\ Teodoro Aragona \\ Rossana Domanico \\ Aldo Garozzo}

Department of Otolaryngology, Head and Neck Surgery, University of Catanzaro, Catanzaro, Italy
Correspondence: Eugenia Allegra Unità Operativa di Otorinolaringoiatria, Università "Magna Graecia” di Catanzaro, Viale Europa, Località Germaneto, 88100 Catanzaro, Italy

Tel $+3996|3647| 30$

Fax $+3996|3647| 3 \mid$

Email eualle@unicz.it
Background and objective: Supracricoid partial laryngectomy (SCL) was introduced as an organ preservation procedure for treating selected early laryngeal cancer. However, the recovery of the voice after SCL may result in different degrees of dysphonia. To improve the functional recovery and quality of the voice, we realized a modified supracricoid laryngectomy (MSCL) using sternohyoid muscles for neoglottic reconstruction in selected patients affected by T1b-T2 laryngeal cancer. In this study, we evaluate the quality of life (QoL) in patients treated by SCL and MSCL.

Methods: The quality of life (QoL) evaluation was undertaken using the Italian version of the European Organization for Research and Treatment of Cancer Quality of Life Questionnaire.

Results: The overall QoL, assessed with European Organization for Research and Treatment of Cancer Quality of Life Questionnaire C30, was better in patients treated with MSCL than in those treated with SCL. The better QoL correlates with the highest response scores to the questions on the relative global functioning scales in patients treated with MSCL.

Conclusion: The new surgical technique has improved the QoL of patients with early laryngeal cancer, with improved communication ability achieved. Reconstruction of neocords in MSCL improves speech function in comparison to SCL, and patients experience less discomfort and achieve an almost normal communication performance.

Keywords: supracricoid laryngectomy, quality of life, laryngeal carcinoma, EORTC QLQ

\section{Introduction}

Organ preservation laryngeal surgery has been defined as a combination of procedures that remove a portion of larynx while maintaining the physiological functions of speech, swallowing, and respiration without compromising local control and cure rate or requiring the need for a permanent tracheostoma. ${ }^{1}$

Supracricoid partial laryngectomy (SCL) was introduced as an organ preservation procedure for treating selected early laryngeal cancer. The preservation of the cricoarytenoid unit, either in part or in its entirety, represents the most important functional condition for good global functional recovery of the neolarynx. ${ }^{2,3}$

In SCL, reconstruction of neolarynx is performed using three sutures that secure the cricoids tightly to the hyoid bone. However, SCL can result in different degrees of dysphonia. ${ }^{4-8}$

To improve the functional recovery and the quality of the voice, we realized a modified supracricoid laryngectomy (MSCL) using sternohyoid muscles for neoglottic reconstruction in selected patients affected by $\mathrm{T} 1 \mathrm{~b}-\mathrm{T} 2$ laryngeal cancer. ${ }^{9}$ 
Only patients with mobile vocal folds and no evidence of thyroid cartilage invasion were eligible for SCL.

MSCL was performed, as previously described, ${ }^{9}$ using sternohyoid muscles on both sides. Muscles were isolated and detached from hyoid bone and fashioned into a tubular shape. This was then placed on the free margin of the cricoids bilaterally and anchored to the vocal apophysis of the arytenoids. ${ }^{9} 10$

The new technique produces a more efficient lateralmedial movement of the arytenoids and recreates the anatomic conditions for physiologic glottis movement, with better results in terms of functional recovery and quality of voice in comparison to traditional SCL. ${ }^{10}$

As an organ preservation treatment modality is deemed successful when the patient achieves the best functional recovery possible, in this study, we evaluated the quality of life (QoL) in patients treated by SCL and MSCL.

QoL was evaluated using the Italian version of the European Organization for Research and Treatment of Cancer Quality of Life Questionnaire (EORTC QLQ).

\section{Materials and methods}

This study was performed between 2006 and 2010 and the study population comprised 80 consecutive untreated patients affected by $\mathrm{T} 1 \mathrm{~b}-\mathrm{T} 2$ laryngeal carcinoma. A total of 34 patients opted for endoscopic surgery or radiotherapy and were excluded from the study; 22 patients were treated by SCL and 24 by MSCL.

MSCL was performed as previously described using sternohyoid muscles on both sides to realize the neocords. ${ }^{9}$ Each sternohyoid muscle was fashioned into a tubular shape, placed on the free margin of the cricoids bilaterally, and anchored to the vocal apophysis of the arytenoids (Figure 1).

The Institutional Review Board of the Magna Graecia University of Catanzaro, Italy, approved the protocol. All patients were informed of the therapeutic alternatives (including endoscopic surgery and nonsurgical treatment), benefits, and complications before giving informed consent for treatment by MSCL.

Selected patients had to be eligible for SCL, with the chance of preserving both arytenoids.

All patients were followed up 1 month after surgery and every 3 months thereafter for 5 years, in our outpatient clinic with laryngoscopy, stroboscopy, and neck palpation. Furthermore, neck ultrasound and chest radiography were performed every 6 months.

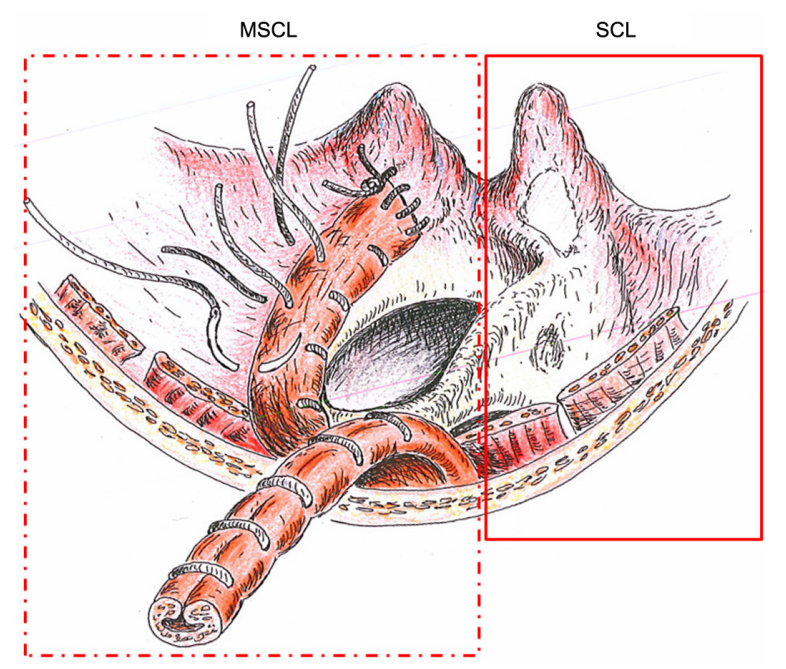

Figure I Illustration of cricoids and arytenoids after modified supracricoid laryngectomy (MSCL) and supracricoid partial laryngectomy $(\mathrm{SCL})$. Left (box with dashed border) MSCL: the sternohyoid muscles of both sides are detached from the hyoid bone, leaving their inferior vascularization, then they are fashioned into a singular tubular shape and placed on the free margin of the cricoids. Finally, they are anchored to the vocal apophysis of the arytenoids for neocord building. Right (box with solid border) SCL: the free margin of the cricoids and arytenoids are covered by redundant mucosa.

\section{QoL evaluation}

QoL was evaluated using the Italian version of the EORTC QLQ. ${ }^{11}$ The core questionnaire (QLQ-C30) is a cancerspecific questionnaire that measures health-related QoL for cancer patients validated for head and neck cancer. It includes 30 items, comprising six functional scales (physical, role, cognitive, emotional and social functioning, and global QoL), three symptom scales (fatigue, pain, and emesis), and six individual items (dyspnea, insomnia, appetite, constipation, diarrhea, and financial position).

Patients also completed the EORTC QoL questionnaire module validated for head and neck cancer (QLQ-H\&N35). ${ }^{12,13}$ This includes 35 items ideated to assess symptom-specific items and contains seven multiple-item scales (pain, swallowing, senses, speech, social eating, social contact, and sexuality) and ten single items (teeth, dry mouth, coughing, opening mouth, sticky saliva, nutritional supplements, feeding tubes, painkillers, weight loss, and weight gain).

All scales were scored on 0-100-point rating scales. On the functional scales higher scores represent better functioning while on the symptom and individual item scales higher scores indicate greater difficulties.

The questionnaires were administered 6 months after the operation.

Patients filled out the questionnaire on their own in a separate room during follow-up visits. If they had difficulty 
in answering questions, a member of the study team was available to provide appropriate assistance.

\section{Statistical analysis}

Analysis was undertaken using MedCalc Software (v 9.0; MedCalc Software bvba, Ghent, Belgium). Data obtained for all scales and items are described as mean scores and standard deviations. A paired-sample $t$-test was used to calculate the mean difference between paired observations. The Mann-Whitney U test for independent samples was performed to analyze group differences. Tests were two tailed and $P<0.05$ was considered significant.

\section{Results}

All sociodemographic and clinical characteristics are displayed in Table 1.

All 22 patients treated by SCL were male, and their ages ranged from 56 to 78 years (mean 61.3 years). Of these patients, 16 had stage T1b cancer: 14 were treated with cricohyoidoepiglottopexy (CHEP) and two with

Table I Sociodemographic and clinical characteristics of patients submitted to supracricoid partial laryngectomy (SCL) and modified supracricoid laryngectomy (MSCL)

\begin{tabular}{|c|c|c|}
\hline & SCL & MSCL \\
\hline Patients, $\mathrm{n}$ & 22 & 24 \\
\hline \multicolumn{3}{|l|}{ Age } \\
\hline Median, years & 61.3 & 59 \\
\hline Range, years & $56-78$ & $45-76$ \\
\hline \multicolumn{3}{|l|}{ Gender, n (\%) } \\
\hline Male & $22(100)$ & $22(9 \mid .6)$ \\
\hline Female & 0 & $2(8.4)$ \\
\hline \multicolumn{3}{|l|}{ Occupation } \\
\hline Employed, n (\%) & $10(45.5)$ & $18(75)$ \\
\hline Retired, n (\%) & $12(54.5)$ & $6(25)$ \\
\hline \multicolumn{3}{|l|}{ Smoking habits } \\
\hline Smoker, n (\%) & $22(100)$ & $22(9 \mid .6)$ \\
\hline Nonsmoker, n (\%) & 0 & $2(8.4)$ \\
\hline \multicolumn{3}{|l|}{ Alcohol consumption } \\
\hline Drinker, n (\%) & $22(100)$ & $22(9 \mid .6)$ \\
\hline Nondrinker, n (\%) & 0 & $2(8.4)$ \\
\hline \multicolumn{3}{|c|}{ Clinical tumor staging, n (\%) } \\
\hline TIbNOMo & $16(72.7)$ & I4 (58.4) \\
\hline T2NoMo & $6(27.3)$ & $10(4 \mid .6)$ \\
\hline \multicolumn{3}{|c|}{ Surgical reconstruction, n (\%) } \\
\hline CHEP & $16(72.7)$ & 17 (70.8) \\
\hline $\mathrm{CHP}$ & $6(27.3)$ & $7(29.2)$ \\
\hline \multicolumn{3}{|c|}{ Follow-up period, months } \\
\hline Mean & 42.5 & 33.5 \\
\hline Range & $35-60$ & $8-46$ \\
\hline
\end{tabular}

Abbreviations: CHEP, cricohyoidoepiglottopexy; CHP, cricohyoidopexy. cricohyoidopexy (CHP). Six patients had stage T2 cancer: two were treated with CHEP and four with CHP.

MSCL was used to treat 22 males and two females (age range 45-76 years [mean 59 years]). Of these patients, 14 had stage T1b cancer: 11 were treated with CHEP and three with CHP. Ten patients had stage T2 cancer: six were treated with CHEP and four with CHP.

All patients had mobile vocal folds, and the arytenoids were preserved. All patients were N0 clinically and radiologically. There were no differences in general condition or in smoking habits and alcohol consumption between the two groups, and female patients in the second group were nonsmokers and nondrinkers.

Mean follow-up time was 42.5 months (range 35-60 months) and 33.5 months (range 8-46 months) $(P>0.05)$ for patients treated by SCL and by MSCL, respectively. Patients treated by SCL were mainly treated at the beginning of the introduction of the MSCL technique.

One patient treated by SCL died from a second tumor (gastric cancer) 47 months after the operation, and one patient treated by MSCL had a recurrence after 38 months. This patient received a total laryngectomy and remains alive at time of writing. Of all employed patients, six out of ten $(60 \%)$ patients treated by SCL and 15 out of $18(83.3 \%)$ patients treated by MSCL returned to work; the remaining patients were already retired.

\section{QoL}

No patient had difficulties in understanding or answering the questions and the mean time for completing both questionnaires was 15 minutes (range 10-20 minutes).

Table 2 reports the descriptive statistics for the core questionnaire (QLQ-C30) of the patients who submitted to SCL and MSCL. There were differences between the two groups in terms of physical functioning, role functioning, and social functioning, with patients treated by SCL having greater difficulties. A significant difference in global health status between the two groups confirmed this trend $(P<0.05)$.

Comparison of the mean scores of QLQ-C30 for the functioning scale, global health status, and symptoms scales between the two treatment groups revealed a significant difference for global health status, which was better in patients treated by MSCL than in those treated by SCL $(78.6 \pm 16.9$ and $61.3 \pm 21.8$, respectively; $P=0.04$ ).

The symptom scales and items in the QLQ-C30 had low scores (SCL, $13.0 \pm 4.5$; MSCL, $14.5 \pm 8.2 ; P=0.57$ ) and 
Table 2 European Organization for Research and Treatment of Cancer Quality of Life Questionnaire (EORTC QLQ)-C30 descriptive statistics for supracricoid partial laryngectomy (SCL) and modified supracricoid laryngectomy (MSCL)

\begin{tabular}{|c|c|c|c|c|c|}
\hline \multirow[t]{2}{*}{ EORTC QLQ-C30 } & \multicolumn{2}{|l|}{ SCL } & \multicolumn{2}{|l|}{ MSCL } & \multirow[t]{2}{*}{$P$} \\
\hline & Mean & SD & Mean & SD & \\
\hline Physical functioning & 79.4 & 22.6 & 87.1 & 16.4 & 0.15 \\
\hline Role functioning & 81.9 & 33.7 & 92.7 & 16.3 & 0.52 \\
\hline Emotional functioning & 71.4 & 20.8 & 72.4 & 24.9 & 0.87 \\
\hline Cognitive functioning & 85.6 & 15.3 & 87.1 & 15.6 & 0.59 \\
\hline Social functioning & 76.0 & 18.7 & 80.7 & 19.9 & 0.27 \\
\hline Global health status & 61.3 & 21.8 & 78.6 & 16.9 & $0.04 *$ \\
\hline \multicolumn{6}{|l|}{ (QoL) } \\
\hline Fatigue & 18.0 & 30.7 & 22.6 & 20.0 & 0.23 \\
\hline Nausea and vomiting & 9.0 & 17.2 & 6.1 & 13.9 & 0.73 \\
\hline Pain & 12.1 & 22.4 & 14.8 & 24.1 & 0.75 \\
\hline Dyspnea & 3.0 & 10.0 & 1.8 & 7.8 & 0.76 \\
\hline Insomnia & 9.0 & 21.5 & 27.7 & 40.0 & 0.21 \\
\hline Appetite loss & 3.0 & 10.0 & 9.2 & 19.1 & 0.39 \\
\hline Constipation & 15.1 & 31.1 & 7.4 & 18.2 & 0.50 \\
\hline Diarrhea & 30.2 & 34.8 & 22.2 & 30.2 & 0.53 \\
\hline Financial difficulties & 21.1 & 30.7 & 12.9 & 23.2 & 0.52 \\
\hline
\end{tabular}

Notes: *statistically significant.

Abbreviations: QoL, quality of life; SD, standard deviation.

neither of the two groups reported great problems in these areas (Figure 2).

Table 3 illustrates the descriptive statistics for the QLQ-H\&N35 questionnaire for both groups of patients. Significantly better speech and social contact scores were reported for patients treated by MSCL when compared with patients treated by SCL $(P<0.02)$.

Eight of 22 patients who were treated by SCL (36.3\%) and 16 of 24 patients treated by MSCL (66.6\%) responded "not at all" to the question concerning problems talking to other people and on the telephone on the speech scale. Neither of the two treatment groups reported significant eating problems. All patients were self-powered and did not use nutritional supplements. The social eating scale returned a very low score for both groups (mean 13.3 and 10.2, for patients treated by SCL and MSCL, respectively).

Low scores on the other scales were related to eating (such as swallowing), the senses, the teeth, opening the mouth, and sticky saliva.

\section{Discussion}

In the present study, health-related QoL after open-organ preservation surgery was evaluated to emphasize improvements allowed by the new surgical technique of MSCL. The QLQ-C30 and QLQ-H\&N35 questionnaires are easy to administer and analyze modification of the patient's life following surgery, which inevitably affects QoL. In our study, the overall QoL assessed using EORTC QLQ-C30 was better in patients treated with MSCL than in those treated with SCL. The better QoL seems to correlate with the highest response scores to the questions on the relative global functioning scales in patients treated with MSCL.

Quality of voice and swallowing recovery after laryngeal surgery mainly affect QoL. Patients treated by MSCL had higher scores in two questions related to (1) talking on the phone and to others and (2) hoarseness, which asked patients to score their perception of the sound of their voice.

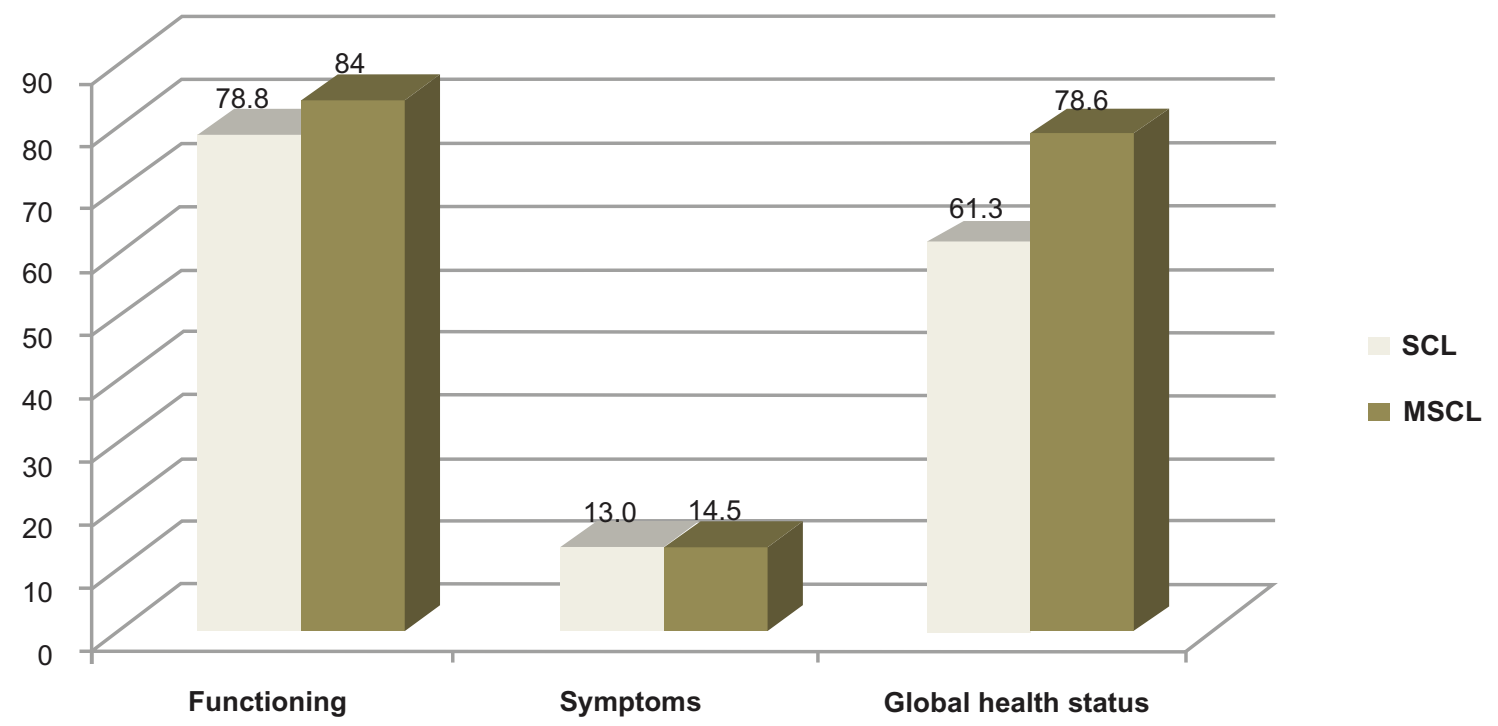

Figure 2 European Organization for Research and Treatment of Cancer Quality of Life Questionnaire C30 comparison of patients treated by supracricoid partial laryngectomy (SCL) or modified supracricoid laryngectomy (MSCL). Comparison of functioning scales $(P=0.05)$, symptoms $(P=0.57)$, and global health status $(P=0.04)$ mean scores between the two treatment groups. 
Table 3 European Organization for Research and Treatment of Cancer Quality of Life Questionnaire (EORTC QLQ)-H\&N35 descriptive statistics for supracricoid partial laryngectomy (SCL) and modified supracricoid laryngectomy (MSCL)

\begin{tabular}{|c|c|c|c|c|c|}
\hline \multirow[t]{2}{*}{ EORTC QLQ-H\&N35 } & \multicolumn{2}{|l|}{ SCL } & \multicolumn{2}{|l|}{ MSCL } & \multirow[t]{2}{*}{$P$} \\
\hline & Mean & SD & Mean & SD & \\
\hline Pain & 9.9 & 8.5 & 9.2 & 16.4 & 0.81 \\
\hline Swallowing & 20.8 & 13.7 & 14.1 & 16.3 & 0.25 \\
\hline Senses & 9.9 & 16.0 & 10.7 & 24.9 & 0.77 \\
\hline Speech & 57.7 & 23.7 & 36.4 & 15.6 & $0.02 *$ \\
\hline Social eating & 13.3 & 15.8 & 10.2 & 19.9 & 0.24 \\
\hline Social contact & 28.7 & 28.6 & 10.1 & 16.9 & $0.02 *$ \\
\hline Sexuality & 23.3 & 41.7 & 23.5 & 20.0 & 0.77 \\
\hline Teeth & 6.6 & 14.0 & 5.8 & 13.9 & 0.65 \\
\hline Opening mouth & 13.3 & 28.0 & 0.0 & 24.1 & 0.39 \\
\hline Dry mouth & 13.3 & 32.2 & 23.5 & 7.8 & 0.11 \\
\hline Sticky saliva & 15.6 & 33.5 & 19.9 & 40.0 & 0.47 \\
\hline Coughed & 16.6 & 17.5 & 16.1 & 19.1 & 1.00 \\
\hline Felt ill & 0.0 & 0.0 & 3.9 & 18.2 & 0.35 \\
\hline Painkillers & 11.7 & 33.2 & 10.0 & 30.2 & 0.60 \\
\hline Nutritional supplements & 0.0 & 0.0 & 0.0 & 0.0 & - \\
\hline Feeding tube & 0.0 & 0.0 & 0.0 & 0.0 & - \\
\hline Weight loss & 0.0 & 0.0 & 15.2 & 16.4 & 0.13 \\
\hline Weight gain & 20.0 & 42.1 & 17.6 & 16.3 & 0.89 \\
\hline
\end{tabular}

Notes: *statistically significant.

Abbreviation: SD, standard deviation.

As previously reported, patients treated by MSCL have a significantly lower perception of voice handicap in comparison to those treated by SCL and this is supported by the voice handicap index (VHI) mean values, especially on the physical subscale. ${ }^{10}$

The global data on eating in public, as assessed by the EORTC QLQ-H\&N35, show no significant differences between the two groups. All patients were fed naturally, did not use nutritional supplements, and were able to chew and swallow food.

In addition, there were no significant differences in terms of mean age and cognitive function. Analyzing these two questionnaires, the scale of symptoms suggests that patients in neither of the two groups felt sick, which correlates with the scores they recorded on the physical function scale.

The available data in the literature on the assessment of QoL using the EORTC QLQ questionnaire in patients treated for laryngeal cancer are mainly related to patients who undergo total laryngectomy. Furthermore, in most of those studies, the questionnaires were administered after a follow-up period ranging from 40 months to 6 years. ${ }^{14-16}$

Singer et al reported data from a German group of patients treated with SCL. ${ }^{12}$ The questionnaires were administered after a longer follow-up period than that used in our study. They found no difference between total laryngectomy and partial laryngectomy in the patients' perceived swallowing and speech functioning. This confirms that the time of administration of the questionnaires affects patients' selfassessment of their new condition. Furthermore, patients who underwent total laryngectomy consider voice impairment a minor consequence of their treatment, given their survival. ${ }^{13}$ Many factors can influence a patient's self-assessment of these functions, including psychosocial traits and cultural and ethnic background.

Singer et al raised the issue of the module EORTC QLQH\&N35 not being sensitive enough to differentiate speech difficulties and that additional symptom scales must be administered. ${ }^{12}$ They referred essentially to the "hoarseness" question for laryngectomized patients and suggested including the item "communicating in noisy environments." We agree with this suggestion, as it could improve understanding of the communication problems of patients undergoing organ preservation laryngeal surgery.

In our study, we analyzed the QoL in a highly selective group of patients who had received organ preservation laryngeal surgery for $\mathrm{T} 1 \mathrm{~b}-\mathrm{T} 2$ laryngeal cancer. Both techniques (MSCL and SCL) result in good QoL and good functional recovery, confirming the validity of organ preservation surgery as a treatment modality for selected cases of laryngeal carcinomas. However, we believe that MSCL has improved the QoL of patients by resulting in an improved communication ability in comparison to that resulting from SCL.

In the Italian population, notable for being particularly talkative, communicative ability is reflected in relationships with family and friends and in the work environment and this has a significant impact on the global QoL. This is supported by our results, which show a high number of patients returning to work after surgery.

Moreover, in the context of organ preservation, the EORTC QLQ-C30 and QLQ-H\&N-35 questionnaires also allow comparison between different therapeutic strategies. ${ }^{14}$ However, data reported in the literature on the assessment of QoL through the EORTC QLQ usually compare total laryngectomy with organ preservation strategies, such as chemo-radiation therapies.

To date, to the best of our knowledge, no comparison of QoL in patients with early larynx cancer treated by open-organ preservation surgery, endoscopic surgery, and chemo-radiation therapies has been done using the EORTC questionnaire. Future studies could examine differences in QoL and establish new therapeutic strategies.

\section{Conclusion}

SCL represents a modality of organ preservation surgery that provides good QoL in selected patients with early 
laryngeal cancer. MSCL introduces the reconstruction of the neocords, resulting in improved speech function, which is perceived by patients as a better communication ability. This technique is limited, however, in that it is restricted for use only in patients with T1b-T2 laryngeal cancer for preservation of both arytenoids.

\section{Disclosure}

The authors report no conflicts of interest in this work.

\section{References}

1. Akbas Y, Demireller A. Oncologic and functional results of supracricoid partial laryngectomy with cricohyoidopexy. Otolaryngol Head Neck Surg. 2005;132(5):783-787.

2. Di Nicola V, Fiorella ML, Spinelli DA, Fiorella R. Acoustic analysis of voice in patients treated by reconstructive subtotal laryngectomy. Evaluation and critical review. Acta Otorhinolaryngol Ital. 2006;26(2): 59-68.

3. Laccourreye O, Cauchois R, Menard M, Brasnu D, Laccourreye H. Deglutition and partial supracricoid laryngectomies. Ann Otolaryngol Chir Cervicofac. 1992;109(2):73-75. French.

4. Guerrier B, Lallemant JG, Balmigère G, Bonnet P, Arnoux B. Our experience in reconstructive surgery in glottic cancers. Ann Otolaryngol Chir Cervicofac. 1987;104(3):175-179. French.

5. Torrejano G, Guimarães I. Voice quality after supracricoid laryngectomy and total laryngectomy with insertion of voice prosthesis. $J$ Voice. 2009;23(2):240-246.

6. Crevier-Buchman L, Laccourreye O, Monfrais-Pfauwadel MC, Menard M, Jouffre V, Brasnu D. Computerized evaluation of acoustic parameters of voice and speech after partial supracricoid laryngectomy with cricohyoidoepiglottopexy. Ann Otolaryngol Chir Cervicofac. 1994;111(7):397-401. French.
7. Crevier-BuchmanL,LaccourreyeO,WuytsFL, Monfrais-PfauwadelMC, Pillot C, Brasnu D. Comparison and evolution of perceptual and acoustic characteristics of voice after supracricoid partial laryngectomy with cricohyoidoepiglottopexy. Acta Otolaryngol. 1998;118(4): 594-599.

8. Bron L, Pasche P, Brossard E, Monnier P, Schweizer V. Functional analysis after supracricoid partial laryngectomy with cricohyoidoepiglottopexy. Laryngoscope. 2002;112(7 Pt 1):1289-1293.

9. Garozzo A, Allegra E, La Boria A, Lombardo N. Modified supracricoid laryngectomy. Otolaryngol Head Neck Surg. 2010;142(1): 137-139. e1.

10. Allegra E, Lombardo N, La Boria A, et al. Quality of voice evaluation in patients treated by supracricoid laryngectomy and modified supracricoid laryngectomy. Otolaryngol Head Neck Surg. 2011;145(5):789-795.

11. Singer $\mathrm{S}$, Wollbrück D, Wulke C, et al. A 12 country field study of the EORTC QLQ-C30 (version 3.0) and the head and neck cancer specific module (EORTC QLQ-H\&N35) in head and neck patients. EORTC Quality of Life Group. Eur J Cancer. 2000;36(14):1796-1807.

12. Singer S, Wollbrück D, Wulke C, et al. Validation of the EORTC QLQC30 and EORTC QLQ-H\&N35 in patients with laryngeal cancer after surgery. Head Neck. 2009;31(1):64-76.

13. Zotti P, Lugli D, Vaccher E, Vidotto G, Franchin G, Barzan L. The EORTC quality of life questionnaire-head and neck 35 in Italian laryngectomized patients. European organization for research and treatment of cancer. Qual Life Res. 2000;9(10):1147-1153.

14. Hanna E, Sherman A, Cash D, et al. Quality of life for patients following total laryngectomy vs chemoradiation for laryngeal preservation. Arch Otolaryngol Head Neck Surg. 2004;130(7):875-879.

15. Nalbadian M, Nikolaidis V, Nikolaou A, Themelis C, Kouloulas A, Vital V. Psychometric properties of the EORTC head and neck-specific quality of life questionnaire in disease-free Greek patients with cancer of pharynx and larynx. Qual Life Res. 2010;19(5):761-768.

16. Lundström E, Hammarberg B, Munck-Wikland E. Voice handicap and health-related quality of life in laryngectomees: assessments with the use of VHI and EORTC questionnaires. Folia Phoniatr Logop. 2009;61(2):83-92.
Open Access Surgery

\section{Publish your work in this journal}

Open Access Surgery is an international, peer-reviewed, open access journal that focuses on all aspects of surgical procedures and interventions. Patient care around the peri-operative period and patient outcomes post surgery are key topics. All grades of surgery from minor cosmetic interventions to major surgical procedures are covered. Novel techniques

Submit your manuscript here: http://www.dovepress.com/open-access-surgery-journal

\section{Dovepress}

and the utilization of new instruments and materials, including implants and prostheses that optimize outcomes constitute major areas of interest. The manuscript management system is completely online and includes a very quick and fair peer-review system. Visit http://www.dovepress.com/ testimonials.php to read real quotes from published authors. 